Rev. Biol. Neotrop. 10(2): 9-17. 2013

\title{
VALIAÇÃO DO EFEITO MODULADOR DA LEVEDURA DE CERVEJA (SACCHAROMYCES CEREVISIAE M.) SOBRE A GENOTOXICIDADE INDUZIDA PELA DOXORRUBICINA EM DROSOPHILA MELANOGASTER
}

\author{
Victor Borges Rezende \\ Universidade Federal de São Carlos, Laboratório de Genética de Populações e Evolução, \\ São Carlos, São Paulo, Brasil, Email: victorrez85@yahoo.com.br
}

\section{Aline Batista Lins}

Universidade Federal de Goiás, Campus Catalão, Departamento de Ciências Biológicas, Catalão, Goiás, Brasil, Email: alinelins.bio@gmail.com

\section{Alexandre Azenha Alves de Rezende}

Universidade Federal de Uberlândia, Laboratório de Mutagênese, Uberlândia, Minas Gerais, Brasil, Email: aleazenha@yahoo.com.br

\author{
Mário Antônio Spanó \\ Universidade Federal de Uberlândia, Laboratório de Mutagênese, Uberlândia, Minas Gerais, \\ Brasil, Email: maspano@ufu.br
}

\section{Neila Coelho de Sousa}

Universidade Federal de Goiás, Campus Catalão, Laboratório de Genética e Mutagênese, Email: n_coelho7@yahoo.com

\begin{abstract}
Resumo: O teste de mutação e recombinação somática (SMART) em Drosophila melanogaster foi utilizado para avaliar o potencial de genotoxicidade e antigenotoxicidade do produto comercial levedura de cerveja (Saccharomyces cerevisiae). Este composto é uma mistura complexa usada pela população para manter o equilíbrio orgânico por ser rico em vitaminas do complexo B, proteínas, minerais e carboidratos. O SMART foi usado na versão padrão (ST - fêmeas $f r^{3} \times$ machos $m w h$ ) para verificar compostos de ação direta e, na versão alta bioativação (HB - fêmeas $O R R ; f l r^{3} \times$ machos $m w h$ ), para verificar compostos de ação indireta, em três diferentes quantidades de levedura de cerveja $(10,0 \%, 20,0 \%$ ou $40,0 \%$ $\mathrm{p} / \mathrm{p}$ ). A essas quantidades foram adicionadas $1,5 \mathrm{~g}$ de purê de batata e hidratados com $5 \mathrm{ml}$ de água ou doxorrubicina (DXR) $(0,125 \mathrm{mg} / \mathrm{mL})$. As larvas obtidas dos cruzamentos ST e HB foram submetidos ao tratamento crônico e feita a análise das asas dos adultos emergentes. A levedura de cerveja não foi genotóxica em ambos os cruzamentos, porém, possui efeitos moduladores sobre a genotoxicidade da DXR,como também exibiu uma atividade sinergistica. Portanto, são necessárias mais pesquisas para determinar os possíveis riscos que podem estar associados com a exposição de organismos a esta mistura complexa.
\end{abstract}

Palavras chave: Drosophila melanogaster; genotoxicidade e antigenotoxicidade; Levedura de cerveja.

Abstract: The Somatic Mutation and Recombination Test (SMART) in Drosophila melanogaster was used to evaluate the genotoxic and antigenotoxic potential of the commercial product of beer's yeast (Saccharomyces cerevisiae). This compound is a complex misture used by the population to maintain the organic equilibrium once it is rich in B vitamins, protein, minerals and carbohydrates. It was used the SMART standard cross (ST - $f r^{3}$ females $\mathrm{x}$ mwh males), to check the compound direct activity, and in the high bioactivation cross( $\mathrm{HB}-O R R ; f l r^{3}$ females $\mathrm{x} m w h$ males) to check indirect activity of three different beer's yeast concentrations $(10.0 \%, 20.0 \%$ or $40.0 \% \mathrm{w} / \mathrm{w})$. To these amounts were added $1,5 \mathrm{~g}$ de mashed potato and hydrated with $5 \mathrm{~mL}$ of water or doxorubicin (DXR) $(0,125 \mathrm{mg} / \mathrm{mL})$. The larvae 
obtained from ST and HB crosses were submitted to chronic treatment and the analysis of wings of adults emerging was done. Beer's yeast was not genotoxic in both crosses, however showed modulatory effects on the genotoxicity of DXR, furthermore it also demonstrated sinergistic activity. Therefore, further research is necessary to determine the possible risks that could be associated with the exposure of organisms to this complex misture.

KeY wORDs: Beer's yeast; Drosophila melanogaster; genotoxicity and antigenotoxicity.

\section{INTRODUÇÃo}

Levedura é um nome genérico que agrupa uma variedade de fungos, incluindo tanto espécies patógenas para plantas e animais, como espécies de grande utilidade para o homem. Algumas espécies de levedura do gênero Saccharomyces são mais conhecidas por realizarem o processo de fermentação e estarem envolvidas em pesquisas genéticas, sendo um modelo biológico pioneiro para estudar a regulação genética do metabolismo eucariótico (Ljungdahl \& Daignan-Fornier, 2012; Matuo et al., 2012).

o Saccharomyces cerevisiae Meyen, vulgarmente apelidado de levedura de cerveja, é um fungo unicelular, ascomicético e elipsoidal que se multiplica assexuadamente através de brotos ou sexuadamente por esporulação (Pelczar et al., 1980; Pelczar Jr. et al., 2004; Pretorius, 2000). Já o complemento nutricional levedura de cerveja é um composto concentrado de leveduras da espécie Saccharomyces cerevisiae mais populares encontrados no mercado (supermercados, farmácias e drogarias) e é utilizado pela população para a manutenção do equilíbrio orgânico. A levedura de cerveja é rica em termos alimentares por ser fonte natural de vitaminas do complexo $B$ (B1 e B3), proteínas, sais minerais (ferro, cálcio, sódio, potássio e fósforo) e carboidratos e é responsável pelo processo de fermentação do açúcar de cereais como o arroz, cevada, malte e trigo, obtendo-se, assim, as bebidas alcoólicas (Applegate \& Grivetti, 1997; Porter, 1995). Também, é muito utilizada em academias visando à busca ou manutenção de um corpo esteticamente perfeito e saudável. Esta preocupação contemporânea com a aparência é advinda de uma pressão da sociedade e da mídia em relação ao corpo padrão (Santos \& Santos, 2002; Stricker, 2002; Queiroz et al., 2009).

É sabido que a levedura de cerveja atua no organismo por meio de suas vitaminas, proteínas, carboidratos e sais minerais e, por isso, suas funções estão associadas à manutenção de seu equilíbrio (Nelson \& Cox, 2006). O complemento alimentar levedura de cerveja é indicado, segundo o fabricante Prolev do Brasil (Abreu e Lima, Pernambuco, Brasil), contra a fadiga e o estresse, para o fortalecimento e embelezamento das unhas e cabelos, para o aumento da imunidade celular, auxilia na regulação do metabolismo do açúcar no organismo e para a prevenção e trata- mento da deficiência de vitaminas do complexo B. Os produtos elaborados com a finalidade de complementar a dieta cotidiana de uma pessoa saudável, que deseja compensar um possível déficit de nutrientes, a fim de alcançar os valores da Dose Diária Recomendada (DDR) não substituem o alimento, não podendo ser utilizado como dieta exclusiva (ANVISA Resolução 19/1999).

Muitos compostos presentes nos alimentos já foram testados quanto à genotoxicidade ou antigenotoxicidade, constatando-se que cerca de um terço de todos os cânceres humanos pode estar relacionado com o hábito alimentar. As evidências na literatura médica demonstraram uma clara associação entre dieta e câncer (Antunes et al, 1998), portanto diante da ampla utilização da levedura de cerveja, faz-se necessária uma investigação quanto à sua genotoxicidade ou antigenotoxicidade.

A mosca das frutas, Drosophila melanogaster, é um excelente organismo experimental para estudar mecanismos moleculares de genotoxicidade e de antigenotoxicidade, com respostas relevantes e que podem ser extrapoladas com um índice de acerto de $92 \%$ para humanos (Cunha, 2001).

O teste de mutação e recombinação somática- SMART (Somatic Mutation and Recombination Test) é um teste rápido, in vivo, versátil que utiliza a $D$. melanogaster como organismo experimental na detecção de eventos genotóxicos induzidos por compostos puros simples e misturas complexas. É um teste ideal para estudos de genotoxicidade e para investigações sobre efeitos moduladores de genotoxicidade (Graf et al., 1998). Este teste possibilita a detecção simultânea de mutação gênica (mutação de ponto), mutação cromossômica (quebra cromossômica, não-disjunção, e translocação) e/ou recombinação mitótica (especialmente os eventos relacionados com recombinação homóloga) (Graf et al., 1984; Würgler \& Vogel, 1986).

As linhagens de $D$. melanogaster denominadas $\mathrm{mwh}$, $\mathrm{flr}^{3}$ e ORR; flr $^{3}$ garantem dois tipos de cruzamentos: padrão (fêmeas flr $^{3} \times$ machos mwh) - que detecta genotoxinas de ação direta, pois utiliza linhagens portadoras de nível basal de enzimas de metabolização do tipo citocromo P-450; e o de alta capacidade de bioativação metabólica (fêmeas ORR; $f^{3}{ }^{3} \mathrm{x}$ machos mwh)- que detecta genotoxinas de ação indireta, e utiliza linhagens com alto nível constitutivo de enzimas citocromo P-450, o que Ihes confere alta 
atividade de metabolização, capacitando ativação enzimática de promutágenos e procarcinógenos (Graf \& Singer, 1992; Graf \& van Schaik 1992; Guzmán-Rincón \& Graf, 1995).

A segurança e a qualidade dos produtos naturais comercializados devem ser asseguradas diretamente por meio de pesquisas, farmacovigilância, controle regulatório e melhor comunicação entre pacientes e profissionais da saúde (Zhou et al., 2004).

O objetivo deste trabalho foi investigar a ação genotóxica e antigenotóxica da levedura de cerveja (Prolev do Brasil), por meio do teste SMART.

\section{Material e Métodos}

\section{COMPOSTOS QUÍMICOS E MEIOS DE CULTURA}

O complemento alimentar, levedura de cerveja, foi obtido da indústria Prolev do Brasil (Abreu e Lima, Pernambuco, Brasil). A informação nutricional contida no frasco do produto é descrita a partir de uma porção de $6 \mathrm{~g}$, ou 12 comprimidos, dos seguintes compostos: $2,1 \mathrm{~g}$ de carboidratos; 2,4 $\mathrm{g}$ de proteínas; $0,21 \mathrm{~g}$ de gorduras totais; $0,16 \mathrm{mg}$ de cálcio; 0,36 mg de ferro; $6,3 \mathrm{mg}$ de sódio; 0,16 mg de vitamina B1; 3 mg de niacina; $54 \mathrm{mg}$ de fósforo e $103 \mathrm{mg}$ de potássio; levedura de cerveja (S. cerevisiae), antiumectante, fosfato tricálcico e maltodextrina.

O cloridrato de doxorrubicina (Adriblastina ${ }^{\circledR} \mathrm{RD}$, $10 \mathrm{mg}$, Lt: 4Y40G4, local BC11) foi adquirido do laboratório Pharmacia Italia S.p.A., Milão - Itália, importado e distribuído por Pharmacia Brasil LTDA., São Paulo, SP, Brasil e dissolvido em água ultra pura na ausência de luz. A água ultra pura (18.2 $\mathrm{M} \Omega$ ), usada como controle negativo para o presente teste, foi obtida de um sistema MilliQ (Millipore, Vimodrone, Milan, Itália).

O Purê de batata em flocos foi obtido de Yoki Alimentos S.A. - São Bernardo do Campo, SP, Brasil. Todas as soluções foram preparadas no momento de uso.

\section{LiNHAGENS DE DROSOPHILA MELANOGASTER}

Três linhagens diferentes da mosca foram utilizadas: (i) machos mwh $(m w h / m w h)$; (ii) fê-

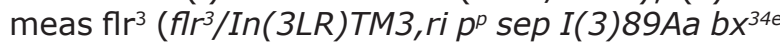
e $\left.B d^{S}\right)$; e (iii) fêmeas ORR; flr $^{3}\left(O R R ; f^{3} r^{3} / \operatorname{In}(3 L R)\right.$ $T M 3$, ri p pep I(3)89Aa bx ${ }^{34 e}$ e $\left.B d^{S}\right)$. Para maiores detalhes sobre os marcadores genéticos ver Lindsley \& Zimm (1992).

\section{Cruzamentos do SMART}

Foram empregados dois tipos de cruzamentos: o padrão (standard - ST), que utiliza machos mwh e fêmeas flr $^{3}$ (Graf et al., 1989), caracterizado por nível basal de metabolização de enzimas citocromo P-450; e o aprimorado (Hight
Bioactivation - HB), que utiliza machos mwh e fêmeas ORR; flr ${ }^{3}$ (Graf \& van Schaik, 1992), caracterizado por alto nível de metabolização de enzimas citocromo P-450 (Hällström \& Blanck, 1985; Saner et al., 2006). A última linhagem possui os cromossomos 1 e 2 da linhagem Oregon(R)R resistente ao DDT (Dapkus \& Merrel, 1977). Tanto o cruzamento ST quanto o HB produzem dois tipos de descendentes: moscas trans-heterozigotas marcadas ( $\left.\mathrm{MH}-m w h+/+f l r^{3}\right)$ e moscas heterozigotas balanceadas $\left(\mathrm{BH}-m w h+/+T M 3, B d^{S}\right)$. Os descendentes BH são distinguíveis fenotipicamente por suas asas serrilhadas.

\section{Procedimento eXPerimental}

Após dois dias de cruzamento, os casais foram transferidos para um meio de oviposição descrito por Graf et al., 1984, usado para a obtenção de larvas de terceiro estágio, constituído de uma base de ágar-ágar a 3\% e recoberto por uma camada de fermento biológico suplementado com sacarose. Os casais permaneceram neste meio por $8 \mathrm{~h}$ e foram descartados.

Para a avaliação da genotoxicidade, após 72 \pm 4 h do início da oviposição, as larvas foram coletadas, lavadas em água corrente e transferidas para frascos contendo $1,5 \mathrm{~g}$ de purê de batata, as diferentes quantidades de levedura de cerveja $(10 \%, 20 \%$ ou $40 \% \mathrm{p} / \mathrm{p})$ e adicionados $5 \mathrm{~mL}$ de água ultra pura. Para o controle positivo de genotoxicidade foram adicionados $5 \mathrm{~mL}$ de DXR a $0,123 \mathrm{mg} / \mathrm{mL}$ ao purê de batata e, para o controle negativo, $5 \mathrm{~mL}$ de água ultra pura. Para a avaliação da antigenotoxicidade, o mesmo procedimento e as mesmas massas foram utilizadas, porém as diferentes concentrações foram hidratadas com $5 \mathrm{~mL}$ de DXR $(0,125 \mathrm{mg} / \mathrm{mL})$. As larvas foram mantidas nestes frascos até a emergência dos adultos (tratamento crônico), a uma temperatura de $25^{\circ} \mathrm{C}$ e umidade relativa de $65 \%$. Após esse período, as moscas foram eterizadas até a morte e estocadas em etanol $70 \%$.

Para montagem do material, as asas foram retiradas e fixadas em lâminas de vidro com solução de Faure e recobertas com lamínula. A análise foi realizada em microscópio óptico, em aumento de 400x. Foram observados e registrados as frequências dos pelos mutantes, os tipos de manchas (mwh e/ou flr), o tamanho e a posição em que se encontravam na asa.

\section{AnÁLISE estATÍSTICA}

Para avaliar os possíveis efeitos genotóxicos a frequência de manchas por mosca em cada série tratada foi comparada ao controle negativo (água) e para a avaliação dos possíveis efeitos antigenotóxicos a frequência de manchas por mosca foi comparada ao controle positivo (DXR).

A análise estatística foi realizada utilizando o programa de computador SMART que usa o teste 
Qui-quadrado para proporções e permite um procedimento de múltipla decisão (Frei \& Würgler, 1988). Os diagnósticos positivos obtidos (para o total do número de manchas) foram confirmados pelos testes não paramétricos $U$-teste de Wilcoxon e Mann \& Whitney (Frei \& Würgler, 1995). As percentagens de inibição ou indução de levedura de cerveja foram calculadas de acordo com Abraham (1994) que é expressa por: [(DXR puro - levedo e DXR)/DXR puro] x 100.

As diferentes quantidades $(10 \%, 20 \%$, ou $40 \% \mathrm{p} / \mathrm{p}$ ) do produto comercial levedura de cerveja puro ou em associação com o mutágeno referência DXR $(0,125 \mathrm{mg} / \mathrm{mL})$ foram testadas e repetidas nos cruzamentos padrão (ST) e de alta bioativação (HB). Os controles positivos e negativos também foram incluídos. Como não houve diferença significativa entre os indivíduos testados nas repetições os resultados foram somados.

\section{Resultados e Discussão}

A levedura de cerveja, na forma de produto industrializado, tem sido muito procurada como coadjuvante para o equilíbrio orgânico e para a busca de um corpo estético e fisicamente perfeito, geralmente em academias. Isso se deve à sua composição química, que é uma mistura complexa de vitaminas do complexo $B$, proteínas e sais minerais (Applegate \& Grivetti, 1997; Porter, 1995), fosfato tricálcico e maltodextrina.

O produto comercial levedo de cerveja nas concentrações de $10 \%, 20 \%$ ou $40 \%$ p/p, foi testado em duas repetições, nos cruzamentos ST e $\mathrm{HB}$, ambos para a verificação de genotoxicidade e antigenotoxicidade, respectivamente. Os controles negativos (água) e positivos (DXR) foram também incluídos. Como não foram encontradas diferenças significativas entre os resultados de duas repetições dos indivíduos provenientes dos cruzamentos ST e HB, os dados foram somados. Ambos os cruzamentos (ST e HB) produziram dois tipos de indivíduos: moscas trans-heterozigotas marcadas $(\mathrm{MH})\left(\mathrm{mwh}+/+f f r^{3}\right)$ e moscas heterozigotas balanceadas $(\mathrm{BH})\left(+/+T M 3, B d^{S}\right)$, estas últimas podem ser distinguidas fenotipicamente por suas asas serrilhadas.

A Tabela 1 apresenta os resultados observados no cruzamento ST. Para verificar a significância estatística para genotoxicidade, as frequências de manchas mutantes observadas entre as moscas tratadas com levedura de cerveja foram comparadas ao controle negativo e, para a antigenotoxicidade, os resultados foram comparados ao controle positivo (DXR).

TABELA 1. Frequência de manchas mutantes observadas entre os descendentes MH e BH de D. melanogaster do cruzamento padrão (ST), tratados com diferentes concentrações da levedura de cerveja (LC). Número de indivíduos (NI); Manchas simples pequenas (MSP); Mancha simples grande (MSG); Mancha gêmea (MG); Total de manchas (TM); Manchas com clones (MC); Frequência de indução de manchas (FIM); Observado (Obs); Controle corrigido (CC); Recombinação (R); Inibição (Ib); Indução (Id).

\begin{tabular}{|c|c|c|c|c|c|c|c|c|c|c|c|}
\hline \multicolumn{2}{|c|}{$\begin{array}{l}\text { Genótipos e } \\
\text { tratamento }\end{array}$} & \multirow[b]{2}{*}{$\begin{array}{l}\mathrm{NI} \\
(\mathrm{N})\end{array}$} & \multicolumn{4}{|c|}{$\begin{array}{c}\text { Manchas por indivíduo (número de manchas) diagnóstico } \\
\text { estatístico }\end{array}$} & \multicolumn{5}{|c|}{$\begin{array}{l}\text { FIM (por } 10^{5} \text { élulas } \\
\text { por divisão celular) }\end{array}$} \\
\hline $\begin{array}{c}\mathrm{DXR} \\
(\mathrm{mg} / \mathrm{mL})\end{array}$ & $\begin{array}{l}\text { LC } \\
(\%)\end{array}$ & & $\begin{array}{c}\text { MSP } \\
(1-2 \text { cells })^{\mathrm{b}} \\
m=2\end{array}$ & $\begin{array}{c}\text { MSG } \\
(>2 \text { cells })^{\mathrm{b}} \\
m=5\end{array}$ & $\begin{array}{c}\text { MG } \\
m=5\end{array}$ & $\begin{array}{c}\text { TM } \\
m=2\end{array}$ & $\begin{array}{c}\mathrm{MC} \\
\mathrm{mwh}^{\mathrm{b}} \\
(n)\end{array}$ & Obs & CC & $\begin{array}{c}\mathrm{R} \\
(\%)\end{array}$ & $\begin{array}{c}\mathrm{Ib}^{d}(\downarrow) \text { ou } \\
\mathrm{Id}^{d}(\uparrow) \\
(\%)\end{array}$ \\
\hline \multicolumn{12}{|l|}{$m w h / f f r^{3}$} \\
\hline 0 & 0 & 30 & 0,30 (09) & $0,03(01)$ & $0,00(00)$ & $0,33(10)$ & 9 & 0,68 & & & \\
\hline 0 & 10 & 20 & $0,20(04)$ & $0,00(00)$ & $0,00(00)$ & $0,20(04)$ & 4 & 0,41 & $-0,27$ & & \\
\hline 0 & 20 & 20 & $0,10(0,2)$ & $0,00(00)$ & $0,00(00)$ & $0,10(0,2)$ & 2 & 0,20 & $-0,48$ & & \\
\hline 0 & 40 & 20 & $0,15(0,3)$ & $0,00(00)$ & $0,00(00)$ & $0,15(0,3)$ & 3 & 0,31 & $-0,38$ & & \\
\hline 0.125 & 0 & 20 & $7,70(154)+$ & $8,4(168)+$ & $8,2(164)+$ & $24,3(486)+$ & 437 & 44,26 & 43,58 & 95,88 & \\
\hline 0.125 & 10 & 20 & $13,4(268) *$ & $11,2(224) *$ & $10,35(207)^{*}$ & $34,95(699) *$ & 672 & 68,34 & 67,66 & 95,42 & $55,25 \uparrow$ \\
\hline 0.125 & 20 & 20 & $10,4(208) *$ & $7,6(152)$ & $8,55(171)$ & $26,55(531)$ & 510 & 51,74 & 51,06 & & \\
\hline 0.125 & 40 & 20 & $6,2(124) *$ & $6,0(120) *$ & $6,0(120) *$ & $18,2(364) *$ & 337 & 34,02 & 33,34 & 96,98 & 23,49 \\
\hline \multicolumn{12}{|l|}{ mwhTM3 3} \\
\hline 0 & 0 & 20 & $0,20(04)$ & $0,00(0)$ & $f$ & $0,20(04)$ & 4 & 0,41 & & & \\
\hline 0.125 & 0 & 20 & $0,65(13)+$ & $0,35(07)+$ & & $1,00(19)+$ & 19 & 1,95 & 1,54 & & \\
\hline 0.125 & 10 & 20 & $1,40(28)^{*}$ & $0,20(04)$ & & $1,60(32)^{*}$ & 32 & 3,28 & 2,87 & & \\
\hline 0.125 & 40 & 20 & $0,40(08)$ & $0,15(03)$ & & $0,55(11)$ & 11 & 1,13 & 0,72 & & \\
\hline
\end{tabular}


CONTINUAÇÃO TABELA 1 - Indivíduos MH (mwh/flr3) e BH (mwh/TM3) foram avaliados. ${ }^{a}$ Diagnóstico estatístico de acordo com Frei \& Würgler (1995). U-test, níveis de probabilidade bilateral: +, p $\leq 0.05$ vs. controle não tratado; ${ }^{*}, \mathrm{p} \leq 0.05$ vs. apenas DXR. ${ }^{\mathrm{b}}$ Considerando clones $m w$ h de manchas $m w h$ simples e gêmeas. ${ }^{c}$ Frequência de formação de clones: clones/indivíduos/48.800 células (sem correção de tamanho). ${ }^{\mathrm{d}}$ Calculado como [somente DXR - (DXR + levedo de cerveja)/somente DXR] x 100, de acordo com Abraham (1994). ${ }^{\mathrm{e}}$ Incluindo manchas simples flr 3 raras. ${ }^{\mathrm{f}}$ Somente manchas simples podem ser observadas em indivíduos $\mathrm{BH}$.

No experimento do teste SMART, a DXR produziu indução de genotoxicidade estatisticamente significante em todas as categorias de manchas nos cruzamentos ST e HB (Tabelas 1 e 2). Esses resultados estão de acordo com Costa et al. 2010, Lehmann et al. (2003), Pereira et al. (2008) e Sousa et al. (2009). Os autores também demonstraram que DXR é preferencialmente um agente indutor de recombinação homóloga. Esses resultados indicam que o teste transcorreu dentro do esperado.

Ainda, como a DXR atua como mutágeno de ação direta verificada pelos cruzamentos ST e HB, respectivamente (Lehmann et al., 2003), nossos resultados demonstram que a levedura de cerveja atua sobre os efeitos desse antibiótico, também, de forma direta.

De acordo com a Tabela 1 no cruzamento ST não foram encontradas diferenças significativas nas frequências de manchas mutantes observadas entre as moscas tratadas em todas as concentrações de levedura de cerveja sem associação com a DXR e o controle negativo. Esses resultados indicam que a levedura de cerveja não possui atividade genotóxica (mutagênica e/ ou recombinogênica) de ação direta, onde os indivíduos testados possuem nível basal de enzimas de metabolização citocromo P-450, ou seja, não necessita de ativação metabólica (Graf et al., 1984).

Também, na Tabela 1, no que se refere ao co-tratamento (diferentes quantidades do produto associadas à DXR), na concentração de $20 \%$ da levedura de cerveja, não foram encontradas diferenças significativas no conjunto de manchas simples grandes (MSG), manchas gêmeas (MG) e total de manchas (TM) quando comparadas à DXR isoladamente. Porém, na concentração de $10 \%$ houve um aumento estatisticamente significativo no número de manchas, revelando uma indução de genotoxicidade pela levedura de cerveja de $55,25 \%$ na frequência de manchas, demonstrando um efeito sinergístico entre DXR e levedura de cerveja. Em relação à concentração de $40 \%$, foi observado uma diminuição significativa em todas as classes de manchas, apresentando uma fraca inibição de genotoxicidade induzida pela DXR $(23,49 \%)$.

Ainda na Tabela 1 , os descendentes $\mathrm{BH}$ do controle com DXR e de levedura de cerveja $10 \%$ e $40 \%$, foram comparados aos $\mathrm{MH}$ e analisados para verificar a porcentagem de recombinação. Essa análise revelou uma porcentagem de 95$97 \%$ de recombinação. Esses resultados demonstram que a levedura de cerveja não alterou a porcentagem de recombinação induzida pela DXR. Nos descendentes BH houve aumento na frequência de manchas devido à mutação e aberração cromossômica, pois devido às múltiplas inversões do cromossomo TM3 não há indução de eventos recombinogênicos.

Assim, pode-se dizer que no cruzamento ST, a levedura de cerveja $10 \%$ apresentou um efeito sinergístico com a DXR e, em $20 \%$, um diagnóstico não significativo. Esses resultados demonstram um baixo poder de ação do composto, mas indicam que deve ser considerado seu efeito potencializador sobre a ação genotóxica da DXR. Ainda, no cruzamento ST, embora a levedura de cerveja $40 \%$ apresente um fraco efeito inibidor, não podemos interpretar como efeito antigenotóxico ou protetor. Segundo Zeiger (2006), cada efeito antimutagênico aparente pode ser resultado da razão de toxicidade e não antimutagenicidade. Neste experimento foi observada uma baixa toxicidade verificada pela diminuição do número de sobreviventes ( 10\%).

A Tabela 2 sumariza os resultados para do cruzamento HB. Esses resultados para a genotoxicidade apresentaram-se negativos em todas as concentrações, indicando que a levedura de cerveja não é um composto de ação indireta e, por isso, não necessita de ativação metabólica (Graf \& Singer, 1992; Graf \& van Schaik, 1992; Guzmán-Rincón \& Graf, 1995). 
TABELA 2. Frequência de manchas mutantes observadas entre os descendentes MH e BH de D. melanogaster do cruzamento de alta bioativação (HB), tratados com diferentes concentrações da levedura de cerveja (LC). Número de indivíduos (NI); Manchas simples pequenas (MSP); Mancha simples grande (MSG); Mancha gêmea (MG); Total de manchas (TM); Manchas com clones (MC); Frequência de indução de manchas (FIM); Observado (Obs); Controle corrigido (CC); Recombinação (R); Indução (Id).

\begin{tabular}{|c|c|c|c|c|c|c|c|c|c|c|c|}
\hline \multicolumn{3}{|c|}{$\begin{array}{l}\text { Genótipos e } \\
\text { tratamento }\end{array}$} & \multicolumn{4}{|c|}{$\begin{array}{l}\text { Manchas por indivíduo (número de manchas) } \\
\text { diagnosticoestatístico }\end{array}$} & & \multicolumn{4}{|c|}{$\begin{array}{l}\text { FIM (por } 10^{5} \text { células por } \\
\text { divisão celular) }\end{array}$} \\
\hline $\begin{array}{c}\text { DXR } \\
(\mathrm{mg} / \mathrm{mL})\end{array}$ & $\begin{array}{l}\text { LC } \\
(\%)\end{array}$ & $\begin{array}{l}\mathrm{NI} \\
(\mathrm{N})\end{array}$ & $\begin{array}{c}\text { MSP } \\
(1-2 \text { cells })^{\mathrm{b}} \\
m=2\end{array}$ & $\begin{array}{c}\text { MSG } \\
(>2 \text { cells })^{b} \\
m=5\end{array}$ & $\begin{array}{l}M G \\
m=5\end{array}$ & $\begin{array}{c}\mathrm{TM} \\
m=2\end{array}$ & $\begin{array}{l}\mathrm{MC} \\
\mathrm{mwh}^{\mathrm{b}} \\
(n)\end{array}$ & Obs & $\mathrm{CC}$ & $\begin{array}{c}\mathrm{R} \\
(\%)\end{array}$ & $\begin{array}{l}I d^{d} \\
(\%)\end{array}$ \\
\hline \multicolumn{12}{|l|}{$m w h / f l r^{3}$} \\
\hline 0 & 0 & 20 & $0,65(13)$ & $0,15(03)$ & $0,00(00)$ & $0.75(15)$ & 15 & 1,54 & & & \\
\hline 0 & 10 & 20 & $0,30(06)$ & $0,00(00)$ & $0,00(00)$ & $0,30(06)$ & 6 & 0,61 & $-0,92$ & & \\
\hline 0 & 20 & 20 & $0,25(05)$ & $0,05(01)$ & $0,00(00)$ & $0,30(06)$ & 5 & 0,61 & $-0,92$ & & \\
\hline 0 & 40 & 20 & $0,55(11)$ & $0,15(03)$ & $0,05(01)$ & $0,70(14)$ & 14 & 1,43 & $-0,10$ & & \\
\hline 0.125 & 0 & 20 & $7,35(147)+$ & $10,75(215)+$ & $8,35(167)+$ & $26,45(529)+$ & 454 & 44,98 & 43,44 & 94,51 & \\
\hline 0.125 & 10 & 20 & $12,5(250) *$ & $14,75(295) *$ & $15,00(300) *$ & $42,25(845) *$ & 733 & 73,57 & 72,03 & 94,08 & 70,42 \\
\hline 0.125 & 20 & 20 & $17,6(352) *$ & $9,70(194)$ & $9,75(195)$ & $37,05(741) *$ & 702 & 70,39 & 68,85 & 92,44 & 58,49 \\
\hline 0.125 & 40 & 20 & $11,95(239) *$ & $6,00(120) *$ & $6,55(131) *$ & $24,50(490)$ & 468 & 46,41 & 45,07 & & \\
\hline
\end{tabular}

mwhTM3

$\begin{array}{ccccc}0 & 0 & 20 & 0,50(10) & 0,20(04) \\ 0.125 & 0 & 20 & 1,10(22)+ & 0,35(07) \\ 0.125 & 10 & 20 & 1,80(36) * & 0,70(14) \\ 0.125 & 20 & 20 & 2,45(49) * & 0,35(07)\end{array}$

$\begin{array}{cccc}0,70(14) & 14 & 1,43 & \\ 1,45(29)+ & 29 & 2,97 & 1,54 \\ 2,50(50) * & 50 & 5,12 & 3,69 \\ 2,80(56) * & 56 & 5,74 & 4,30\end{array}$

Indivíduos MH (mwh/flr3) e BH (mwh/TM3) foram avaliados. ${ }^{a}$ Diagnóstico estatístico de acordo com Frei \& Würgler (1995). U-test, níveis de probabilidade bilateral:,$+ \mathrm{p} \leq 0.05$ vs. controle não tratado; *, $\mathrm{p} \leq 0.05$ vs. apenas DXR. ${ }^{b}$ Considerando clones $m w$ h de manchas $m w$ h simples e gêmeas. ${ }^{c}$ Frequência de formação de clones: clones/indivíduos/48.800 células (sem correção de tamanho). ${ }^{\mathrm{d}}$ Calculado como [somente DXR - (DXR + levedo de cerveja)/somente DXR] x 100, de acordo com Abraham (1994). ${ }^{\mathrm{e}}$ Incluindo manchas simples flr3 raras. ${ }^{\mathrm{f}}$ Somente manchas simples podem ser observadas em indivíduos $\mathrm{BH}$.

Por outro lado, nos resultados para o co-tratamento (antigenotoxicidade) também houve indução nas frequências do número total de manchas de $70,42 \%$ e $58,49 \%$ nas concentrações de $10 \%$ e $20 \%$, respectivamente. A concentração de $40 \%$ de levedura de cerveja apresentou resultado estatisticamente não significativo (Tabela 2). Estes resultados mostram efeitos sinergísticos da DXR + levedura de cerveja $10 \%(70,42 \%)$ e $20 \%(58,49 \%)$ e sem efeito significante em $40 \%$. Como esses produtos são compostos por misturas complexas, eles podem exercer sua ação separadamente ou sinergisticamente (Romero-Jiménez et al., 2005).

Ainda, na Tabela 2, a análise dos descendentes $\mathrm{BH}$, nas concentrações de $10 \%$ e $20 \%$, demonstrou um aumento na frequência de manchas devido à mutação e aberração cromossômica, pois esses descendentes não possuem eventos de recombinação.

Constituintes alimentares podem ser metabolizados pelo citocromo P-450 sendo transformados em metabólitos não tóxicos e excretados, mas a formação de metabólitos tóxicos é possível. Dados da literatura indicam que alguns constituintes da dieta podem formar intermediários reativos capazes de inibir de vários citocromos P-450 (Zhou et al., 2004).

Os mecanismos pelos quais a levedura de cerveja utilizou para interagir com a DXR nos permite inferir que seus constituintes podem interagir com o sistema de enzimas que participam da detoxificação de DXR, conduzindo ao aumento da genotoxicidade da DXR. Resultados similares 
foram descritos com ácido tânico em associação com mitomycina $C$, metil metano sulfonato e mostarda nitrogenada em células somáticas de D. melanogaster (Lehmann et al., 2000).

Foram analisados os indivíduos $\mathrm{BH}$ dos cruzamentos ST e HB, os quais foram obtidos resultados positivos, para a verificação da porcentagem de eventos de mutação e recombinação mitótica (tabela 1 e 2). Os indivíduos BH possuem múltiplas inversões que bloqueiam os processos da recombinação e, portanto, permitem quantificar a contribuição real da recombinação, quando os resultados são comparados aos obtidos para os indivíduos trans-heterozigotos (Graf et al., 1984).

Extratos de plantas medicinais contêm misturas complexas de milhares de componentes que podem exercer sua ação separadamente ou em conjunto (Cai et al., 2004; Romero-Jiménez et al., 2005). Já vem sendo estudada a provável relação tóxica das vitaminas no organismo. As vitaminas possuem mecanismos de ação ainda não totalmente desvendados pelo homem e alguns estudos têm avaliado seus efeitos tóxicos associando-os a estresses oxidativos, mutações e ao câncer (Fragiorge et al., 2007). Porém, é necessária uma análise detalhada da interação desses constituintes além de uma análise individual de cada um desses para que se possa afirmar algo a respeito, já que as vitaminas que constituem o composto são lipossolúveis e seu excesso, excretado pelo organismo (Nelson \& Cox, 2006).

Como verificado, o teste SMART é de alta eficiência na detecção de agentes mutagênicos e recombinogênicos e está bem validado com um número estimado de mais de 400 diferentes substâncias químicas ou misturas complexas (Graf et al., 1998; Pantaleão et al., 2007; Sarikaya \& Akir, 2005, Sousa et al., 2009). Assim, podemos sugerir que a levedura de cerveja (S. cerevisiae) do fabricante Prolev do Brasil de modo geral não possui atividade genotóxica de ação direta e indireta e possui uma fraca atividade moduladora sobre a genotoxicidade da DXR, como também apresentou uma ação sinergística quando avaliada pelo teste SMART em Drosophila melanogaster. Porém, outros testes de genotoxicidade e antigenotoxicidade se fazem necessários para melhor avaliação do produto.

Trabalhos similares, onde a levedura de cerveja ou seus compostos poderiam ter sido submetidos a testes de antimutagenicidade não foram encontrados na literatura científica, aumentando a importância destes resultados e, ao mesmo tempo, deixando o assunto aberto para novas indagações.

\section{Agradecimentos}

Ao Conselho Nacional de Desenvolvimento Científico e Tecnológico (CNPq), Coordenação de Aperfeiçoamento de Pessoal de Nível Superior (CAPES), Fundação de Amparo à Pesquisa do Estado de Minas Gerais (FAPEMIG), Universidade
Federal de Uberlândia (UFU) e Universidade Federal de Goiás - Campus Catalão (UFG-CAC) o agradecimento pelo financiamento e apoio.

\section{ReferÊNCIAS}

Abraham, S.K. 1994. Antigenotoxicity of coffee in the Drosophila assay for somatic mutation and recombination. Mutagenesis 9:383-386.

Antunes, L.M.G., M.C.P. Araújo, Dias F.L., C.S. Takahashi. 1998. Modulatory effects of curcumin on the chromosomal damage induced by doxorubicin in Chinese hamster ovary cells. Teratog, Carcinog and mutag, v.19. p.1-8.

ANVISA - Agência Nacional De Vigilância Sanitária. 1999. Aprova o regulamento técnico de procedimentos para registro de alimento com alegação de propriedades funcionais e ou de saúde em sua rotulagem. Resolução no 19, de 10 de dezembro.

Applegate, E.A. \& L.E. Grivetti. 1997. Search for the competitive edge: a history of dietary fads and supplements. Journal of Nutricion 127:869-73.

Cai, Y., Q, Luo, M. Sun \& H. Corke. 2004. Antioxidant activity and phenolic compounds of 112 traditional Chinese medicinal plants associated with anticancer. Life Sci 74: 215784.

Costa, W.F., Oliveira, A.B. \& Nepomuceno, J.C. 2010. Genotoxicity of Lapachol evaluated by wing spot test of Drosophila melanogaster. Genet Mol Biol 33:558-63.

Cunha, K.S. 2001. Agentes antineoplásicos como indutores de toxicidade genética em células somáticas de Drosophila melanogaster. 115f. Tese (Doutorado). Universidade Federal do Rio Grande do Sul, Porto Alegre.

Dapkus, J. \& D.J. Merell. 1977. Chromosomal analysis of DDT-resistance in a long-term selected population of Drosophila melanogaster. Genetic 87:685-97.

Fragiorge, E.J., M.A. Spanó \& L.M.G. Antunes. 2007 Modulatory effects of the antioxidant ascorbic acid on the direct genotoxicity of doxorubicin in somatic cells of Drosophila melanogaster. Genet Mol Biol 30:449-55.

Frei, H.\& F.E. Würgler. 1988. Statistical methods to decide whether mutagenicity test data from Drosophila assays indicate a positive, negative, or inconclusive result. Mutat Res 203:297-308. 
Frei, H. \& F.E. Würgler. 1995. Optimal experimental design and sample size for the statistical evaluation of data from somatic mutation and recombination test (SMART) in Drosophila. Mutat Res 334:247-58.

Graf, U., F.E. Würgler, A.J. Katz, J. Frei, H. Juon, C.B. Hall \& P.G. Kale. 1984. Somatic Mutation and Recombination Test in Drosophila melanogaster. Environ Mutagen 6:153-88.

Graf, U., H. Frei, A. Kagi, A.J. Katz \& F.E. Wurgler. 1989. Thirty compounds tested in the Drosophila wing spot test. Mutat Res 222:359-73.

Graf, U. \& D. Singer. 1992. Genotoxicity testing of promutagens in the wing somatic mutation and recombination test in Drosophila melanogaster. R Int Contam Ambient 8: 15-27.

Graf, U., K.A. Suresh, G.R Judith \& E.W. Friedrich. 1998. Drosophila melanogaster. Antigenotoxicity studies in Drosophilas melanogaster. Mut Res 18:203-9.

Graf, U. \& N. van Schaik. 1992. Improved high bioactivation cross for the wing somatic mutation and recombination test in Drosophila melanogaster. Mutat Res 271:59-67.

Guzmán- Rincon, J. \& V. Graf. 1995. Drosophila melanogaster somatic mutation and recombination test as a biomotor. In: Biomotors and Biomarkers a Indicators of Environmental Cahnge, Edity by F. M. Butterworth et al., Phenunn Press p. 169-81.

Hällström, I. \& A. Blanck. 1985. Genetic regulation of th cytochrome P450 system in Drosophila melanogaster. II. Localization of some genes regulating cytochrome P450 activity. Chem Biol Interact 56:157-71.

Lehmann, M., U. Graf, M. L. Reguly, \& H.H. R. Andrade. 2000. Interference of tannic acid on the genotoxicity of mitomycin C, methylmethanesulfonate, and nitrogen mustard in somatic cells of Drosophila melanogaster. Environ Mol Mutagen 36:195-200.

Lehmann, M., A. Franco, K.S.P. Vilar, M.L. Reguly \& H.H.R. Andrade. 2003. Doxorubicin and two of its analogues are preferential inducers of homologous recombination compared with mutational events in somatic cells of Drosophila melanogaster. Mutat Res 539:167-75.

Lindsley, D.L. \& G.G. Zimm. 1992. The Genome of Drosophila melanogaster. Academic Press, San Diego, 1133p.
Ljungdahl, O. \& B. Daignan-Fornier. 2012. Regulation of Amino Acid, Nucleotide, and Phosphate Metabolism in Saccharomyces cerevisiae. Genetics 190:885-929.

Matuo, R., F.G. Sousa, D.G. Soares, D. Bonatto, J. Saffi, A.E. Escargueil, A.K. Larsen \& J.A.P. Henriques. 2012. Saccharomyces cerevisiae as a model system to study the response to anticancer agents. Cancer Chemother Pharmacol.

Nelson, L.D. \& M.M. Cox. 2006. Princípios de Bioquímica. 4rd ed., Sarvier Press, São Paulo.

Pantaleão, S.M., A.V. Alcântara, J.P.H. Alves, L.A. Pavanin, U. Graf, A.A.A. Rezende, B.L.B. Valadares, E.J. Fragiorge, N.C. Souza, Z.R. Guterres \& M.A. Spanó. 2007. Assessing the impact of pollution on the Japaratuba River in Brazil using the Drosophila wing spot test. Environ Mol Mutagen 48:96-105.

Pelczar Jr, M.J., E.C.S. Cham \& N.R. Kried. 2004. Os Principais Grupos de Microrganismos Eucarióticos: Fungos, Algas e Protozoários. In: Microbiologia. Makron Books 1:26075.

Pelczar, M.J., R. REID \& E.C.S. CHAM. 1980. Fungos: As Leveduras. In: Microbiologia. McGraw-Hill 1:345-66.

Pereira, D.G., L.M.G. Antunes, U. Graf \& M.A. Spanó. 2008. Protection by Panax ginseng C.A. Meyer against the genotoxicity of doxorubicin in somatic cells of Drosophila melanogaster. Genet Mol Biol 31:947-55.

Porter, D.V. 1995. Dietary Supplement: Recent Chronology and Legislation. Nut Rev. 53: 31-6.

Pretorious I.S. 2000. Tailoring Wine yeast for the new millenium: novel approaches to the ancient art of winemaking. Yeast v.16, p.675-729.

Queiroz S.F., H.C. Silva, H.P.O. Bezerra, L.K.P. Freitas, H.A. Costa, J.A.M. Oliveira \& M.L.L. Andrade. 2009. Utilização de suplemento alimentar por usuários de academias de ginástica do município de Pau dos Ferros-RN. RBNE v. 3, n. 17.

Romero-Jiménez M,J. Campos-Sánchez, M. Analla, A. Muñoz-Serrano \& A. Alonso-Moraga. 2005. Genotoxicity and antigenotoxicity of some traditional medicinal herbs. Mutat Res 585:147-55. 
Saner, C., B. Weibel, F.E. Würgler \& C. Sengstag. 2006. Metabolism of promutagens catalyzed by Drosophila melanogaster $\mathrm{CY}$ P6A2 enzyme in Saccharomyces cerevisiae. Environ Mol Mutagen 27:46-58.

Santos, M. A.A. \& R.P. Santos. 2002. Uso de Suplementos Alimentares como Forma de Melhorar a Performance nos Programas de Atividade Física em Academias de Ginástica. Rev Paul Educ Fís 16:174-85.

Sarikaya, R. \& Ş. Akir. 2005. Genotoxicity testing of four food preservatives and their combinations in the Drosophila wing spot test. Environ Toxicol Pharmacol 20:424-30.

Sousa, N.C., A.A.A. Rezende, R.M.G. Silva, Z.R. Guterres, U. Graf, W.E. Kerr \& M.A. Spanó. 2009. Modulatory Effects of Tabebuia impetiginosa (Lamiales, Bignoniaceae) on Doxorubicin-induced Somatic Mutation and Recombination in Drosophila melanogaster. Genet Mol Biol 32:382-8.
Stricker, P.R. 2002. Sports training issues for the pediatric athlete. Petriatr Clin 49:793802.

Würgler, F.E. \& E. Vogel. 1986. In vivo Mutagenicity Testing Using Somatic Cells of Drosophila melanogaster. In: DE SERRES, F. J. (ed.). Chemical mutagens, principles and methods for their detection. Plenum Press 10:1-72.

Zeiger, E. 2006. Ilusions of safety: antimutagens can be mutagens, and anticarcinogens can be carcinogens, Mut Res, Chapel Hill 543:191-4.

Zhou, S., H.L. Koh, Y. Gao, Z. Gong, E.J.D. Lee. 2004. Herbal bioactivation: the good, the bad and the ugly. Life Sci 74:935-68.

Recebido em 7.III.2013

Aceito em 15.VIII.2013 